EPJ Web of Conferences 72, 00022 (2014)

DOI: 10.1051 /epjconf / 20147200022

(C) Owned by the authors, published by EDP Sciences, 2014

\title{
Bottomonia: open bottom strong decays and spectrum
}

\author{
E. Santopinto ${ }^{1, a}$ and J. Ferretti ${ }^{1,2}$ \\ ${ }^{1}$ INFN, Sezione di Genova, via Dodecaneso 33, 16146 Genova (Italy) \\ ${ }^{2}$ Instituto de Ciencias Nucleares, Universidad Nacional Autónoma de México, 04510 México DF, México
}

\begin{abstract}
We present our results for the bottomonium spectrum with self energy corrections. The bare masses used in the calculation are computed within Godfrey and Isgur's relativized quark model. We also discuss our results for the open bottom strong decay widths of higher bottomonia in the ${ }^{3} P_{0}$ pair-creation model.
\end{abstract}

\section{Introduction}

The quark model (QM) [1-14] (see also Refs. [15, 16]) is very successful in reproducing the behavior of observables such as the spectrum and the magnetic moments, but it neglects pair-creation effects. These effects give rise to virtual $q \bar{q}-q \bar{q}(q q q-q \bar{q})$ components in the hadron wave function and shifts of the physical mass with respect to the bare mass [17], as already shown by several authors in the baryon [18-22] and meson [23-36] sectors. Some example are the resonance $\Lambda(1405)$, that decays into $\Sigma \pi$, but it is strongly influenced by the nearby $\bar{K} N$ channel [37], and the $f_{0}(980)$, that decays into $\pi \pi$, but behaves remarkably as a $K \bar{K}$ molecule [38]. The continuum coupling effects are also relevant to the study of other observables, such as the importance of the orbital angular momentum in the spin of the proton [39], the flavor asymmetry of the proton [40] and the strange content of the electromagnetic form factors of the nucleon [41]. Important information on mesons are also provided by the decay modes (strong, electromagnetic and weak decays). In particular, the two-body strong decays are transitions to open-flavor final states, where the starting $b \bar{b}$ (or $c \bar{c}$ ) meson decays by $q \bar{q}$ pair-production ( $q=u, d$ or $s$ ), and then it divides into two open-bottom (or open-charm) mesons. The simplest model, describing this phenomenon, is the ${ }^{3} P_{0}$ pair-creation one, in all its possible variants [42-47].

In this contribution, we discuss an application of the unquenched quark model (UQM) [34-36, 39-41] to the calculation of the bottomonium spectrum with self energies corrections [36]. We also briefly discuss our results for the strong decay widths of higher bottomonia [36], computed within a modified version of the ${ }^{3} P_{0}$ paircreation model [34-36].

\section{Self energies in the UQM}

We consider the following Hamiltonian,

$$
H=H_{0}+V \text {, }
$$

\footnotetext{
ae-mail: santopinto@ge.infn.it
}

that is the sum of a first term, $H_{0}$, acting only in the bare meson space, and a second term, $V$, that couples a meson state $|A\rangle$ to the meson-meson continuum $|B C\rangle$. One has to consider a precise form for the two operators of Eq. (1), $H_{0}$ and $V$. We choose the successful relativized QM of Ref. [4] for the bare Hamiltonian $H_{0}$ and the UQM for mesons [34-36], based on a ${ }^{3} P_{0}$-type operator, for $V$.

The dispersive equation, resulting from a nonrelativistic Schrödinger equation, is given by

$$
\Sigma\left(E_{a}\right)=\sum_{B C} \int_{0}^{\infty} q^{2} d q \frac{\left|V_{a, b c}(q)\right|^{2}}{E_{a}-E_{b c}}
$$

and the bare energy $E_{a}$ satisfies:

$$
M_{a}=E_{a}+\Sigma\left(E_{a}\right)
$$

$M_{a}$ is the physical mass of the meson $A$, with self energy $\Sigma\left(E_{a}\right)$; the matrix element $V_{a, b c}$ is the coupling between $|A\rangle$ and $|B C\rangle$, computed within the UQM for mesons of Refs. [34-36]:

$$
V_{a, b c}(q)=\sum_{\ell J}\left\langle B C \vec{q} \ell J\left|T^{\dagger}\right| A\right\rangle
$$

The UQM approach is a generalization of the unitarized quark model by Törnqvist and Zenczykowski [18] (see also Ref. [48]). It is based on a QM, to which $q \bar{q}$ pairs with vacuum quantum numbers are added as a perturbation and where the pair-creation mechanism is inserted at the quark level [34-36, 39-41].

The symbol $T^{\dagger}$ in Eq. (4) stands for the modified ${ }^{3} P_{0}$ quark-antiquark pair-creation operator [34-36, 42, 43], where we introduced an effective pair-creation strength $\gamma_{0}^{\text {eff }}[27,34-36]$ and also a Gaussian quark form factor [34-36, 39-41, 48, 49], $A$ is the meson, $B$ and $C$ represent the intermediate state mesons, and $E_{a}, E_{b}=\sqrt{M_{b}^{2}+q^{2}}$ and $E_{c}=\sqrt{M_{c}^{2}+q^{2}}$ are the corresponding energies, $\vec{q}$ and $\ell$ the relative radial momentum and orbital angular momentum between $B$ and $C$ and $\vec{J}=\vec{J}_{b}+\overrightarrow{J_{c}}+\vec{\ell}$ is the total 
angular momentum. The wave functions of the mesons $A$, $B$ and $C$ are written as harmonic oscillator wave functions, depending on a single oscillator parameter $\alpha=0.5 \mathrm{GeV}$. In Eq. (2), one has to sum over the possible intermediate states $|B C\rangle$. These meson-meson channels have quantum numbers $J_{b c}$ and $\ell$ coupled to the total angular momentum of the initial state $|A\rangle . E_{b c}=E_{b}+E_{c}$ is the total energy of the channel $B C$, calculated in the rest frame.

The values of the pair-creation model's parameters, used to compute the strong decays of Sec. 3 and the vertices $\left\langle B C \vec{q} \ell J\left|T^{\dagger}\right| A\right\rangle$ of Eq. (4), are reported in Ref. [36]. The value of $\gamma_{0}$ is fitted to the experimental $\Upsilon(4 S) \rightarrow B \bar{B}$ strong decay width [50]; the other values are taken from the literature [34-36].

\section{Open bottom strong decays in the ${ }^{3} P_{0}$ pair-creation model}

Here, we discuss our results for the open bottom strong decay widths of higher bottomonia [36]. The decay widths are given by [34-36, 42, 46, 47]

$$
\Gamma_{A \rightarrow B C}=\Phi_{A \rightarrow B C}\left(q_{0}\right) \sum_{\ell, J}\left|\left\langle B C \vec{q}_{0} \ell J\left|T^{\dagger}\right| A\right\rangle\right|^{2},
$$

where $\Phi_{A \rightarrow B C}\left(q_{0}\right)$ is the relativistic phase space factor [34$36,42,46,47]$,

$$
\Phi_{A \rightarrow B C}=2 \pi q_{0} \frac{E_{b}\left(q_{0}\right) E_{c}\left(q_{0}\right)}{M_{a}},
$$

depending on the relative momentum $q_{0}$ between $B$ and $C$ and on the energies of the two intermediate state mesons, $E_{b}=\sqrt{M_{b}^{2}+q_{0}^{2}}$ and $E_{c}=\sqrt{M_{c}^{2}+q_{0}^{2}}$. The experimental values of the meson masses $M_{a}, M_{b}$ and $M_{c}$ are taken from the PDG [50] and Ref. [51], while for still unobserved states we use Godfrey and Isgur's model predictions, obtained with the values of the model's parameters of Ref. [36].

Finally, in Table 1 we compare some of our results of Ref. [36] to the experimental data [50, 52].

\section{Bare and self energy calculation of $b \bar{b}$ states}

In our study of Ref. [36], the bare energies $E_{a}$ 's of Eqs. (2) and (3) are computed within the relativized QM [4]. At variance with QM calculations, such as those of Ref. [4], we fit the physical masses $M_{a}$ 's of Eq. (3) to the experimental data $[50,51]$, instead of the bare energies $E_{a}$ 's, and therefore the fitting procedure is an iterative one.

After computing the bare masses of the $b \bar{b}$ states, one can calculate the self energies $\Sigma\left(E_{a}\right)$ 's of Eq. (2), summing over a complete set of accessible $\mathrm{SU}_{\mathrm{f}}(5) \otimes \mathrm{SU}_{\text {spin }}(2) 1 S$ intermediate states. If the bare energy of the initial meson $A$ is above the threshold $B C$, the self energy correction due to the channel $B C$ is given by

$$
\begin{aligned}
\Sigma\left(E_{a}\right)(B C) & =\mathcal{P} \int_{M_{b}+M_{c}}^{\infty} \frac{d E_{b c}}{E_{a}-E_{b c}} \frac{q E_{b} E_{c}}{E_{b c}}\left|\left\langle B C \vec{q} \ell J\left|T^{\dagger}\right| A\right\rangle\right|^{2} \\
& +2 \pi i\left\{\frac{q E_{b} E_{c}}{E_{a}}\left|\left\langle B C \vec{q} \ell J\left|T^{\dagger}\right| A\right\rangle\right|^{2}\right\}_{E_{b c}=E_{a}},
\end{aligned}
$$

where the symbol $\mathcal{P}$ stands for a principal part integral.

In Table 2, we show our UQM result for the mass of the $\eta_{b}$ resonance [36]. It is worthwhile noting that the contributions to the self energy due to unphysical heavy $Q \bar{Q}$ pair-creation $(Q=c, b)$ are strongly suppressed, by substituting the pair-creation strength of the ${ }^{3} P_{0}$ model, $\gamma_{0}$, with an effective one, $\gamma_{0}^{\text {eff }}[27,34-36]$.

\section{Continuum components and decay widths in the UQM}

The coupling $T^{\dagger}$ of Eq. (4) gives rise to a continuum component in an initial valence state $|A\rangle$. Its norm, giving the probability that the physical energy eigenstate $\left|\Psi_{A}\right\rangle$ is in the meson-meson continuum, is given by $[29,34]$ :

$$
P_{a}^{\text {sea }}=\sum_{B C \ell J} \int_{0}^{\infty} q^{2} d q \frac{\left|\left\langle B C \vec{q} \ell J\left|T^{\dagger}\right| A\right\rangle\right|^{2}}{\left(E_{a}-E_{b}-E_{c}\right)^{2}},
$$

where one has to sum over virtual channels $B C$. The probability to find $\left|\Psi_{A}\right\rangle$ in its valence component is just $P_{b \bar{b}}=1-P_{a}^{s e a}$.

One can also relate the imaginary part of the self energy of Eq. (7),

$$
\operatorname{IM}\left[\Sigma\left(E_{a} ; B C\right)\right]=2 \pi i\left\{\frac{q E_{b} E_{c}}{E_{a}}\left|\left\langle B C \vec{q} \ell J\left|T^{\dagger}\right| A\right\rangle\right|^{2}\right\}_{E_{b c}=E_{a}}
$$

to the decay width by $[29,34]$

$$
\Gamma_{A \rightarrow B C}=\operatorname{Im}\left[\Sigma\left(E_{a}\right)(B C)\right] \text {. }
$$

In a subsequent paper [53], we intend to compute the continuum components of higher bottomonium states via Eq. (8) and compare our ${ }^{3} P_{0}$ model results [36] for the open bottom strong decay widths to those obtained through Eq. (10). This is particularly interesting in the case of $\chi_{b}(3 P)$ mesons, that may contain important continuum components $[36,53]$.

\section{Conclusions}

In this contribution, we analized the results of an UQM calculation of the spectrum of bottomonia with self energy corrections [36]. The self energies are corrections to the meson masses due to the coupling to the meson-meson continuum.

Even if the self energy corrections to the spectrum of $b \bar{b}$ [36] and $c \bar{c}$ [35] mesons are relatively small, approximately $1-2 \%$ and $2-6 \%$ of the corresponding meson mass, respectively, these continuum-coupling effects can become qualitatively important in the case of suspected non $q \bar{q}$ states, such as the $X(3872)$ [54] or $\chi_{b}(3 P)$ mesons $[55,56]$, that are states close to a meson-meson decay threshold. In particular, in Ref. [35] it is shown that the continuum coupling effects of the $X(3872)$ give rise to $D \bar{D}^{*}$ and $D^{*} \bar{D}^{*}$ components in addition to the $c \bar{c}$ core, while in the case of $\chi_{b}(3 P)$ mesons, these corrections give rise to $B \bar{B}, B \bar{B}^{*}$ and $B^{*} \bar{B}^{*}$ components [36]. 
Table 1. Our results of Ref. [36] for $\Upsilon(4 S), \Upsilon(10860)$ and $\Upsilon(11020)$ are compared to the experimental data [50, 52]. The results are expressed in $\mathrm{MeV}$.

\begin{tabular}{cccc}
\hline State & $\Gamma\left({ }^{3} P_{0}\right)[36]$ & $\Gamma \exp (\mathrm{PDG})[50]$ & $\Gamma \exp ($ BABAR $)[52]$ \\
$\Upsilon(4 S)$ & 21 & $21 \pm 3$ & - \\
$\Upsilon(10860)$ & 71 & $55 \pm 28$ & $74 \pm 4$ \\
$\Upsilon(11020)$ & 36 & $79 \pm 16$ & $37 \pm 3$ \\
\hline
\end{tabular}

Table 2. Self $\left[\Sigma\left(E_{a}\right)\right]$ and bare $\left(E_{a}\right)$ energies of the $\eta_{b}$ state (in MeV), whose sum gives the physical mass $M_{a}$ of the meson [36]. This value is compared to the corresponding experimental data [50]. Columns 2-8 show the contributions to $\Sigma\left(E_{a}\right)$ from various channels $B C$, such as $B \bar{B}^{*}, B^{*} \bar{B}^{*}$ and so on.

\begin{tabular}{cccccccccccc}
\hline State & $B \bar{B}^{*}$ & $B^{*} \bar{B}^{*}$ & $B_{s} \bar{B}_{s}^{*}$ & $B_{s}^{*} \bar{B}_{s}^{*}$ & $B_{c} \bar{B}_{c}^{*}$ & $B_{c}^{*} \bar{B}_{c}^{*}$ & $\Upsilon \Upsilon$ & $\Sigma\left(E_{a}\right)$ & $E_{a}$ & $M_{a}$ & $M_{\text {exp. }}$ \\
& $\bar{B} B^{*}$ & & $\bar{B}_{s} B_{s}^{*}$ & & $\bar{B}_{c} B_{c}^{*}$ & & & & & & \\
\hline$\eta_{b}\left(1^{1} S_{0}\right)$ & -26 & -26 & -5 & -5 & -1 & -1 & 0 & -64 & 9455 & 9391 & 9391 \\
\hline
\end{tabular}

We also discussed our results for the open bottom strong decay widths of $b \bar{b}$ states [36], computed within a modified version of the ${ }^{3} P_{0}$ model [34-36]. These results may be useful to the experimentalists, because the present knowledge of $\eta_{b}$ and $\chi_{b}$ states and, above all, all the other radially and orbitally-excited resonances $(D, F$ and $G$-wave), that we analysed in Ref. [36], is not very good.

Finally, we briefly analysed a possible application of the UQM formalism to the calculation of the continuum components and the two-body strong decay widths of higher bottomonia [53]. We think that this could be especially useful in the case of $\chi_{b}(3 P)$ mesons, that may contain important continuum components [36, 53], being close to $B \bar{B}, B \bar{B}^{*}$ and $B^{*} \bar{B}^{*}$ decay thresholds.

\section{References}

[1] E. Eichten et al., Phys. Rev. Lett. 34, 369 (1975); E. Eichten et al., Phys. Rev. D 17, 3090 (1978); 21, 203 (1980).

[2] N. Isgur and G. Karl, Phys. Rev. D 18, 4187 (1978); 19, 2653 (1979); 20, 1191 (1979).

[3] W. Buchmuller and S. H. H. Tye, Phys. Rev. D 24, 132 (1981).

[4] S. Godfrey and N. Isgur, Phys. Rev. D 32, 189 (1985).

[5] S. Capstick and N. Isgur, Phys. Rev. D 34, 2809 (1986).

[6] F. Iachello, N. C. Mukhopadhyay and L. Zhang, Phys. Rev. D 44, 898 (1991).

[7] W. Lucha, F. F. Schoberl and D. Gromes, Phys. Rept. 200, 127 (1991).

[8] R. Bijker, F. Iachello and A. Leviatan, Annals Phys. 236, 69 (1994).

[9] M. M. Giannini and E. Santopinto, Phys. Rev. C 49, 1258 (1994); M. De Sanctis, M. M. Giannini, E. Santopinto and A. Vassallo, Eur. Phys. J. A 19, 81 (2004); Nucl. Phys. A 755, 294 (2005); Phys. Rev. C 76, 062201 (2007).

[10] L. Y. Glozman, D. O. Riska, Phys. Rept. 268, 263 (1996).
[11] U. Loring, B. C. Metsch and H. R. Petry, Eur. Phys. J. A 10, 395 (2001).

[12] E. Santopinto, Phys. Rev. C 72, 022201 (2005); J. Ferretti, A. Vassallo and E. Santopinto, Phys. Rev. C 83, 065204 (2011); M. De Sanctis, J. Ferretti, E. Santopinto and A. Vassallo, Phys. Rev. C 84, 055201 (2011).

[13] E. Santopinto and G. Galatá, Phys. Rev. C 75, 045206 (2007).

[14] P. Colangelo, F. De Fazio, F. Giannuzzi and S. Nicotri, Phys. Rev. D 86, 054024 (2012).

[15] P. Guo, A. P. Szczepaniak, G. Galata, A. Vassallo and E. Santopinto, Phys. Rev. D 77, 056005 (2008); Phys. Rev. D 78, 056003 (2008).

[16] S. Capstick et al., Eur. Phys. J. A 35, 253 (2008).

[17] C. Patrignani, T. K. Pedlar and J. L. Rosner, arXiv:1212.6552 [hep-ex].

[18] N. A. Törnqvist and P. Zenczykowski, Phys. Rev. D 29, 2139 (1984); Z. Phys. C 30, 83 (1986); P. Zenczykowski, Annals Phys. 169, 453 (1986).

[19] K. G. Horacsek, Y. Iwamura and Y. Nogami, Phys. Rev. D 32, 3001 (1985).

[20] W. Blask, M. G. Huber and B. Metsch, Z. Phys. A 326, 413 (1987).

[21] M. Brack and R. K. Bhaduri, Phys. Rev. D 35, 3451 (1987).

[22] B. Silvestre-Brac and C. Gignoux, Phys. Rev. D 43, 3699 (1991).

[23] S. Ono and N. A. Törnqvist, Z. Phys. C 23, 59 (1984); K. Heikkila et al., Phys. Rev. D 29, 110 (1984); S. Ono et al., Phys. Rev. D 34, 186 (1986).

[24] E. van Beveren and G. Rupp, Phys. Rev. Lett. 91, 012003 (2003); E. van Beveren et al., Phys. Rev. D 74, 037501 (2006); G. Rupp and E. van Beveren, Eur. Phys. J. A 31, 698 (2007).

[25] E. J. Eichten, K. Lane and C. Quigg, Phys. Rev. D 69, 094019 (2004).

[26] D. S. Hwang and D. -W. Kim, Phys. Lett. B 601, 137 (2004).

[27] Y. S. Kalashnikova, Phys. Rev. D 72, 034010 (2005).

[28] M. R. Pennington and D. J. Wilson, Phys. Rev. D 76, 077502 (2007). 
[29] T. Barnes and E. S. Swanson, Phys. Rev. C 77, 055206 (2008).

[30] B. -Q. Li and K. -T. Chao, Phys. Rev. D 79, 094004 (2009).

[31] I. V. Danilkin and Y. .A. Simonov, Phys. Rev. D 81, 074027 (2010).

[32] I. V. Danilkin and Yu. A. Simonov, Phys. Rev. Lett. 105, 102002 (2010).

[33] J. -F. Liu and G. -J. Ding, Eur. Phys. J. C 72, 1981 (2012).

[34] J. Ferretti, G. Galatá, E. Santopinto and A. Vassallo, Phys. Rev. C 86, 015204 (2012).

[35] J. Ferretti, G. Galatá and E. Santopinto, Phys. Rev. C 88, 015207 (2013).

[36] J. Ferretti and E. Santopinto, arXiv:1306.2874 [hep$\mathrm{ph}]$.

[37] R. H. Dalitz and S. F. Tuan, Phys. Rev. Lett. 2, 425 (1959).

[38] S. M. Flatte et al., Phys. Lett. B 38, 232 (1972).

[39] R. Bijker and E. Santopinto, Phys. Rev. C 80, 065210 (2009); E. Santopinto and R. Bijker, Few Body Syst. 44, 95 (2008).

[40] E. Santopinto and R. Bijker, Phys. Rev. C 82, 062202 (2010).

[41] R. Bijker, J. Ferretti and E. Santopinto, Phys. Rev. C 85, 035204 (2012).

[42] L. Micu, Nucl. Phys. B 10, 521 (1969); A. Le Yaouanc et al., Phys. Rev. D 8, 2223 (1973); 9, 1415 (1974); Phys. Lett. B 71, 397 (1977); 72, 57 (1977).
[43] R. Kokoski and N. Isgur, Phys. Rev. D 35, 907 (1987).

[44] W. Roberts, and B. Silvestre-Brac, Few-Body Systems 11, 171 (1992).

[45] S. Capstick and W. Roberts, Phys. Rev. D 47, 1994 (1993); 49, 4570 (1994); 58, 074011 (1998).

[46] E. S. Ackleh, T. Barnes and E. S. Swanson, Phys. Rev. D 54, 6811 (1996).

[47] T. Barnes, S. Godfrey and E. S. Swanson, Phys. Rev. D 72, 054026 (2005).

[48] P. Geiger and N. Isgur, Phys. Rev. D 55, 299 (1997).

[49] P. Geiger and N. Isgur, Phys. Rev. Lett. 67, 1066 (1991); Phys. Rev. D 44, 799 (1991); 47, 5050 (1993).

[50] J. Beringer et al. [Particle Data Group Collaboration], Phys. Rev. D 86, 010001 (2012).

[51] R. Mizuk et al. [Belle Collaboration], Phys. Rev. Lett. 109, 232002 (2012).

[52] B. Aubert et al. [BaBar Collaboration], Phys. Rev. Lett. 102, 012001 (2009).

[53] J. Ferretti and E. Santopinto, in preparation.

[54] S. K. Choi et al. [Belle Collaboration], Phys. Rev. Lett. 91, 262001 (2003); D. Acosta et al. [CDF II Collaboration], Phys. Rev. Lett. 93, 072001 (2004).

[55] G. Aad et al. [ATLAS Collaboration], Phys. Rev. Lett. 108, 152001 (2012).

[56] V. M. Abazov et al. [D0 Collaboration], Phys. Rev. D 86, 031103 (2012). 